\title{
Article
}

\section{Elution Behavior of Carbohydrates for Core-Shell Ion-Exchange Resins with Different Degrees of Cross-Linking in Porous Shell Layer}

\author{
Shun-ichi Mitomo ${ }^{1 *}$, Yukiko Negishi ${ }^{1}$, Toshiki Mutai ${ }^{2}$, and Yutaka Inoue ${ }^{3}$ \\ ${ }^{1}$ Institute of Nutrition Sciences, Kagawa Nutrition University, 3-9-21, Chiyoda, Sakado, Saitama 350-0288, Japan \\ ${ }^{2}$ Institute of Industrial Science, the University of Tokyo, 4-6-1, Komaba, Meguro-ku, Tokyo 153-8505, Japan \\ ${ }^{3}$ Laboratory of Drug Safety Management, Faculty of Pharmacy and Pharmaceutical Science, Josai University, \\ 1-1, Keyakidai, Sakado, Saitama 350-0293, Japan
}

(Manuscript received December 4, 2020; accepted July 6, 2021)

\begin{abstract}
A novel core-shell ion-exchange resin (St-80) having a 20:80 weight ratio of the monomer for the core and the shell was prepared for use in high-performance liquid chromatography (HPLC), and the effect of the degree of cross-linking (10-55\%) of the porous shell on the separation of carbohydrates was examined. A mixed aqueous sample of inositol, glucose, fructose, and sucrose was reasonably separated under strong alkaline conditions ( 0.10 and $0.15 \mathrm{~mol} / \mathrm{L} \mathrm{NaOH}$ eluent) at flow rates of $0.3-0.7 \mathrm{~mL} / \mathrm{min}$. As the degree of cross-linking in the shell portion increased, the retention time of sucrose, which had the longest elution time, decreased. Meanwhile, the theoretical plate number nearly doubled. The retention times obtained for the high degrees of cross-linking ( $40 \%$ and $55 \%)$ in the porous shell were shorter than that of the fully porous resin. The theoretical plate number observed for these resins provided excellent resolution, similar to that of the fully porous resin. Keywords: HPLC, Core-shell ion-exchange resin, Carbohydrates, Retention time, Theoretical plate number
\end{abstract}

\section{Introduction}

In 1992, Kirkland reported the successful synthesis of core-shell resins with a large shell thickness ${ }^{1)}$. However, they only had limited success, and the most recent development in core-shell particles was reported in 2007 by Kirkland ${ }^{2)}$. This new version of the core-shell resin was improved in terms of HPLC. The most used resins in liquid chromatography are inorganic materials, such as silica gel, alumina, and apatite. Various core-shell silica resins, including those whose surfaces are chemically modified with octadecylsilyl groups, have also been developed ${ }^{3-8)}$. However, silica-based resins are not compatible with high-pH conditions because their adsorption ability decreases, and the resins can become soluble in alkaline solutions, limiting the $\mathrm{pH}$ range of the mobile phase.

Styrene-divinylbenzene- and acrylamide-type polymers are frequently used as base materials for organic resins ${ }^{9-12)}$. The physical properties of these polymer resins can be controlled by changing the monomer structure, cross-linking reagent, pore-controlling reagent, and polymerization conditions, allowing the resin to be optimized for various analytes. Furthermore, controlling the particle size and/or surface properties allows the modification in resins for high-speed and high-resolution miniaturization. However, most polymer resins developed to date are of the fully porous type, which has certain limitations for high-speed operation.

The synthesis of core-shell ion-exchange resins can solve this problem because both the shell and core portions are composed of polymers. These resins are considered highly efficient because various functional groups can be introduced into the shell portion. They also provide superior durability under strong alkaline conditions. A core-shell ion-exchange resin fabricated by precipitation polymerization around the core portion $^{13,14)}$ and a latex-type resin that uses styrene as the base ${ }^{15-17)}$ are already commercially available.

A core-shell ion-exchange resin (St-80) prepared using a 20:80 weight ratio of the core and the shell monomers, in addition to a fully porous resin, was prepared by suspension polymerization ${ }^{18-24)}$. The St-80 resin exhibited high functionality, similar to that of the fully porous resin. Furthermore, carbohydrate analysis by HPLC using St-80 demonstrated a

* Corresponding Author E-mail: mitomo@w6.dion.ne.jp 


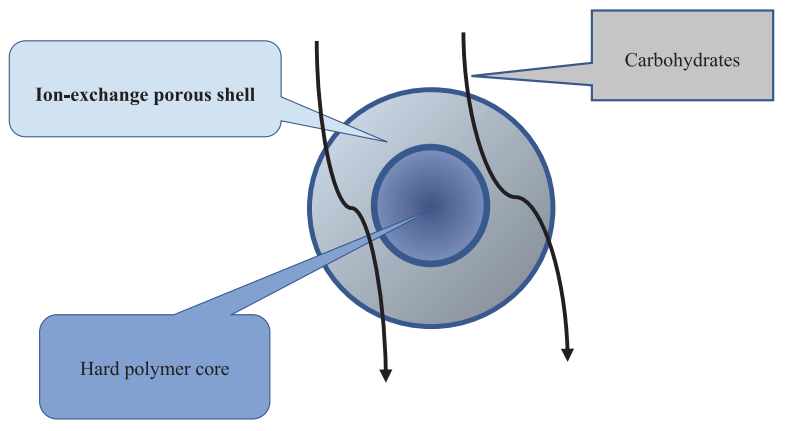

Fig. 1 Structure of the core-shell ion-exchange resin consisting of a hard polymer core and ion-exchange porous shell.

short retention time compared to that obtained using the fully porous resin ${ }^{25-27)}$. Because the retention time of carbohydrates obtained using core-shell ion-exchange resins is short, these are valid tools in the analysis of foods containing carbohydrates.

The two important factors in the development of resins are the thickness ${ }^{28-30)}$ and the change in the degree of cross-linking in the porous layer. Therefore, it is important to clarify the appropriate combination of shell thickness and degree of cross-linking in the porous layer.

In this study, the elution behaviors of carbohydrates of three organic core-shell ion-exchange resins (St-80) with different degrees of cross-linking (10\% (St-80 (10\%)), 40\% (St-80 $(40 \%)$ ), and $55 \%$ (St-80 (55\%)) in the porous layer (Fig. 1) were examined. A short carbohydrate retention time was obtained when the degree of cross-linking of the porous layer was $40 \%$ or $55 \%$. At a fast flow rate of $0.7 \mathrm{~mL} / \mathrm{min}$, these resins provided good separation for each carbohydrate.

\section{Experimental}

\subsection{Reagents}

myo-Inositol, sucrose (Fujifilm Wako Chem. Co.), D(-)-fructose, D(+)-glucose (Kanto Chem. Co.), and sodium hydroxide (Fujifilm Wako Chem. Co.) were obtained from commercial sources. Ultrapure water (ELGA) was used to prepare the eluent and sample solutions. The sample solutions were prepared by sequentially mixing and diluting the stock solutions (500 or $1000 \mathrm{mg} / \mathrm{L}$ ).

\subsection{Conditions for HPLC analysis}

HPLC analysis was performed using a TOA-DKK SU300 instrument equipped with an electrochemical detector (ECD with a gold electrode).

The resin used in this study consisted of a hard polymer core and a porous shell containing functional groups, as shown in Fig. 1. The porous shell portion was synthesized by the reaction of a chloromethylstyrene-divinylbenzene copolymer carrier with a tertiary amine, according to the process described

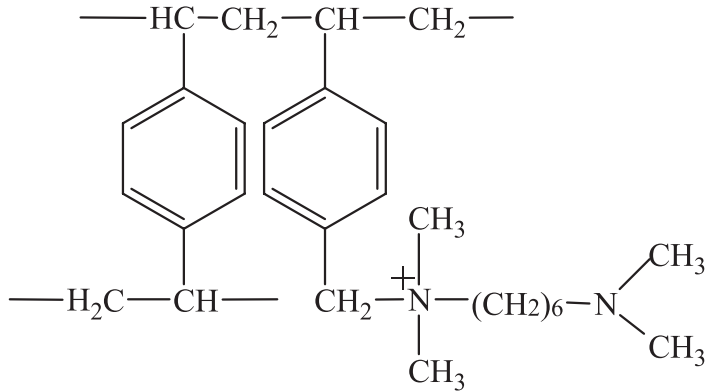

Fig. 2 Chemical structure of porous polymer shell in the ion-exchange resin.

in the literature (Fig. 2) ${ }^{17-20)}$. The thickness of the shell was adjusted by maintaining a constant initial core to shell monomer weight ratio (20:80).

The degree of cross-linking of the porous layer was adjusted by varying the weight ratio of styrene to divinybenzene (45:55 (St-80 (55\%)), 60:40 (St-80 (40\%)), and 90:10 (St-80 $(10 \%) \mathrm{w} / \mathrm{w})$, while retaining the total mass of styrene and divinybenzene. The fully porous resin (55\%) was synthesized by the reaction of a chloromethylstyrene-divinylbenzene copolymer ( $5 \mu \mathrm{m}$ in diameter, divinylbenzene $55 \%$ ) carrier with a tertiary amine. Then, $3 \mathrm{~g}$ of each resin $(\sim 5 \mu \mathrm{m}$ in diameter), St-80 (55\%), St-80 (40\%), St-80 (10\%), and the fully porous resin $(55 \%)$ were mixed with $10 \mathrm{~mL}$ of eluent $(0.10 \mathrm{~mol} / \mathrm{L} \mathrm{NaOH})$. These resins were packed into $4.6 \mathrm{~mm} \times$ $150 \mathrm{~mm}$ I.D. stainless steel column by a conventional slurry packing method using $0.10 \mathrm{~mol} / \mathrm{L} \mathrm{NaOH}$ eluent as the eluent at a constant pressure of $120 \mathrm{~kg} / \mathrm{cm}^{2}$.

The sample solution $(20 \mu \mathrm{L})$ was injected using an AS8020 autosampler (Tosoh) and eluted with either 0.10 or $0.15 \mathrm{~mol} / \mathrm{L}$ $\mathrm{NaOH}$ eluent at room temperature $\left(20-30^{\circ} \mathrm{C}\right)$. The flow rate ranged from 0.3 to $0.7 \mathrm{~mL} / \mathrm{min}$. The theoretical plate number $(N)$ of each carbohydrate in the standard solution was determined using a built-in data processing program.

\section{Results and discussion}

A solution containing inositol, glucose, fructose, and sucrose was used as a standard to evaluate the resins because saccharides are responsible for imparting sweetness to foods such as sweet potatoes and tomatoes. Under all elution conditions, the retention times of these saccharides followed the order of inositol, glucose, fructose, and sucrose.

\subsection{Evaluation of separation properties for St-80 (10\%),} St-80 (40\%), and St-80 (55\%) ion-exchange resins

Chromatograms showing the separation of saccharides in columns packed with St-80 (10\%), St-80 (40\%), and St-80 $(55 \%)$ eluted at a flow rate of $0.5 \mathrm{~mL} / \mathrm{min}$ with $0.10 \mathrm{~mol} / \mathrm{L}$ 

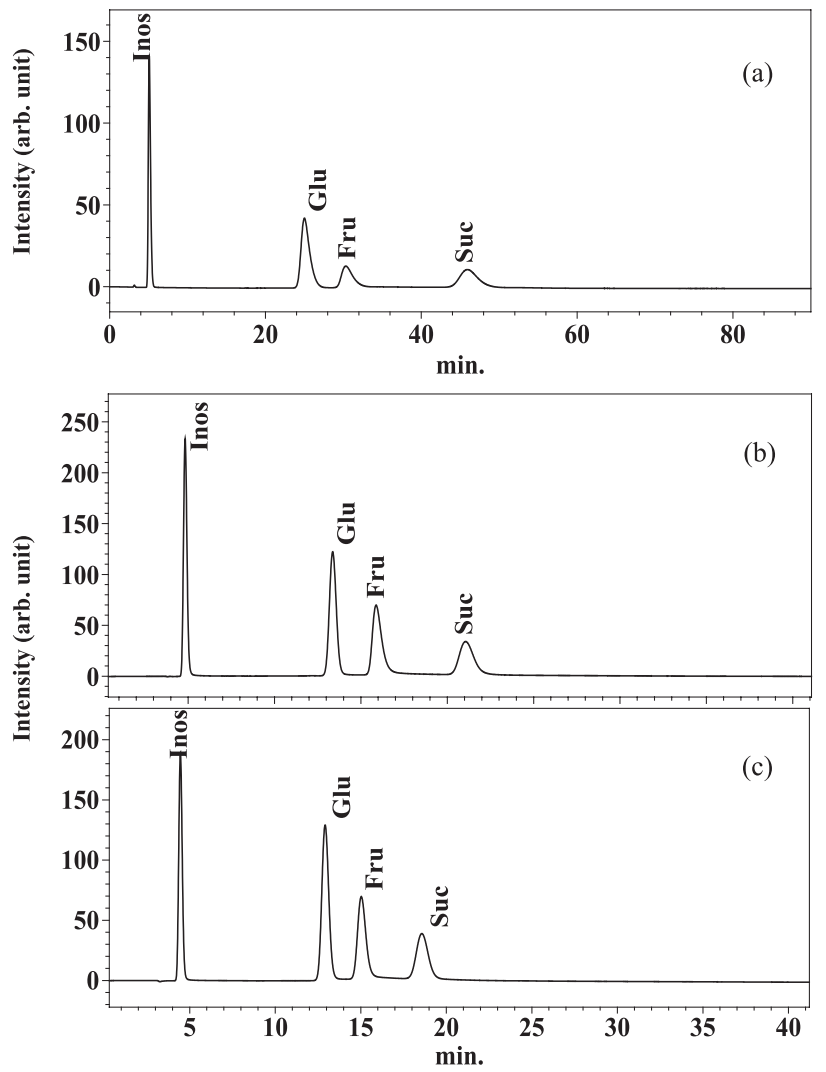

Fig. 3 Chromatograms obtained for separation of inositol, glucose, fructose, and sucrose using St-80 resins of (a) $10 \%$, (b) $40 \%$, and (c) $55 \%$ with $0.10 \mathrm{~mol} / \mathrm{L} \mathrm{NaOH}$ eluent at a flow rate of $0.5 \mathrm{~mL} / \mathrm{min}$.

$\mathrm{NaOH}$ eluent are shown in Figs. 3a-c, respectively.

Chromatograms of St-80 (10\%) at a flow rate of $0.5 \mathrm{~mL} / \mathrm{min}$ with $0.10 \mathrm{~mol} / \mathrm{L} \mathrm{NaOH}$ eluent are shown in Fig. 3a. The retention times of sucrose were approximately 79,46 , and $31 \mathrm{~min}$ at flow rates of $0.3,0.5$, and $0.7 \mathrm{~mL} / \mathrm{min}$, respectively. St-80 (10\%) resin provided good separation of these saccharides under all conditions, even at a low degree of cross-linking $(10 \%)$. At each flow rate, the retention time of St-80 (10\%) was longer than that of St-80 (55\%).

Chromatograms of St-80 (40\%) at a flow rate of $0.5 \mathrm{~mL} /$ min with $0.10 \mathrm{~mol} / \mathrm{L} \mathrm{NaOH}$ eluent are shown in Fig. $\mathbf{3 b}$. The retention times of sucrose were approximately 35,21 , and

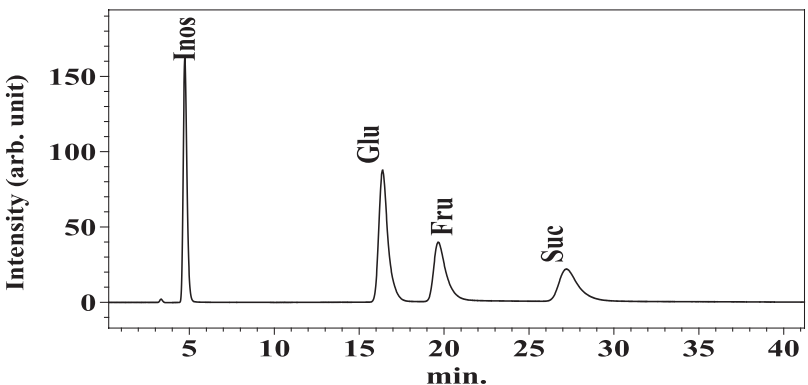

Fig. 4 Chromatograms obtained for separation of inositol, glucose, fructose, and sucrose using a fully porous resin (55\%) with $0.10 \mathrm{~mol} / \mathrm{L} \mathrm{NaOH}$ eluent at a flow rate of $0.5 \mathrm{~mL} / \mathrm{min}$.

$15 \mathrm{~min}$ at flow rates of $0.3,0.5$, and $0.7 \mathrm{~mL} / \mathrm{min}$, respectively. St-80 (40\%) provided good separation of these saccharides, similar to that of resin with $10 \%$ cross-linking at each flow rate.

Chromatograms of St-80 (55\%) at a flow rate of $0.5 \mathrm{~mL}$ / min eluted with $0.10 \mathrm{~mol} / \mathrm{L} \mathrm{NaOH}$ eluent are shown in Fig. 3c. The retention times of sucrose for St-80 (55\%) were approximately 27,19 , and $13 \mathrm{~min}$ at flow rates of $0.3,0.5$, and $0.7 \mathrm{~mL} / \mathrm{min}$, respectively.

The separation properties of these resins were further investigated by determining the resolution between glucose and fructose, which are adjacent peaks in the above chromatograms. As shown in Table 1, elution with $0.10 \mathrm{~mol} / \mathrm{L} \mathrm{NaOH}$ eluent at a flow rate of $0.5 \mathrm{~mL} / \mathrm{min}$ provided resolution of 1.9 , 2.2, and 2.2 for St-80 (10\%), St-80 (40\%), and St-80 (55\%), respectively, and the resolution at a flow rate of $0.7 \mathrm{~mL} /$ min with $0.10 \mathrm{~mol} / \mathrm{L} \mathrm{NaOH}$ eluent were $1.8,2.0$, and 1.9 , respectively, indicating good separation performance even at a high flow rate of $0.7 \mathrm{~mL} / \mathrm{min}^{31}$.

Using the fully porous resin (55\%) and eluting with $0.10 \mathrm{~mol} / \mathrm{L} \mathrm{NaOH}$ eluent, the resolution between glucose and fructose at flow rates of $0.3,0.5$, and $0.7 \mathrm{~mL} / \mathrm{min}$ were 3.0 , 2.6 , and 2.3, respectively. Chromatograms of the fully porous resin with $0.10 \mathrm{~mol} / \mathrm{L} \mathrm{NaOH}$ eluent at a flow rate of $0.5 \mathrm{~mL} /$ min are shown in Fig. 4. The series of St-80 resins exhibited good resolution between glucose and fructose. Thus, these St-80 resins provided good separation performance with the $0.10 \mathrm{~mol} / \mathrm{L} \mathrm{NaOH}$ eluent.

Table 1 Resolution between glucose and fructose using St-80 (10\%, 40\%, and 55\%) resin eluted with either $0.10 \mathrm{~mol} / \mathrm{L} \mathrm{NaOH}$ or $0.15 \mathrm{~mol} / \mathrm{L} \mathrm{NaOH}$ eluent

\begin{tabular}{cccccccc}
\hline $0.10 \mathrm{~mol} / \mathrm{L} \mathrm{NaOH}$ & & Resolution & & $0.15 \mathrm{~mol} / \mathrm{L} \mathrm{NaOH}$ & \multicolumn{3}{c}{ Resolution } \\
\hline Flow rate $(\mathrm{mL} / \mathrm{min})$ & $10 \%$ & $40 \%$ & $55 \%$ & Flow rate $(\mathrm{mL} / \mathrm{min})$ & $10 \%$ & $40 \%$ & $55 \%$ \\
\hline 0.3 & 2.1 & 2.6 & 2.3 & 0.3 & 1.3 & 1.8 & 2.0 \\
0.4 & 2.0 & 2.4 & 2.3 & 0.4 & 1.3 & 1.8 & 2.0 \\
0.5 & 1.9 & 2.2 & 2.2 & 0.5 & 1.3 & 1.6 & 1.9 \\
0.6 & 1.9 & 2.1 & 2.0 & 0.6 & 1.2 & 1.5 & 1.8 \\
0.7 & 1.8 & 2.0 & 1.9 & 0.7 & 1.2 & 1.5 & 1.7 \\
\hline
\end{tabular}




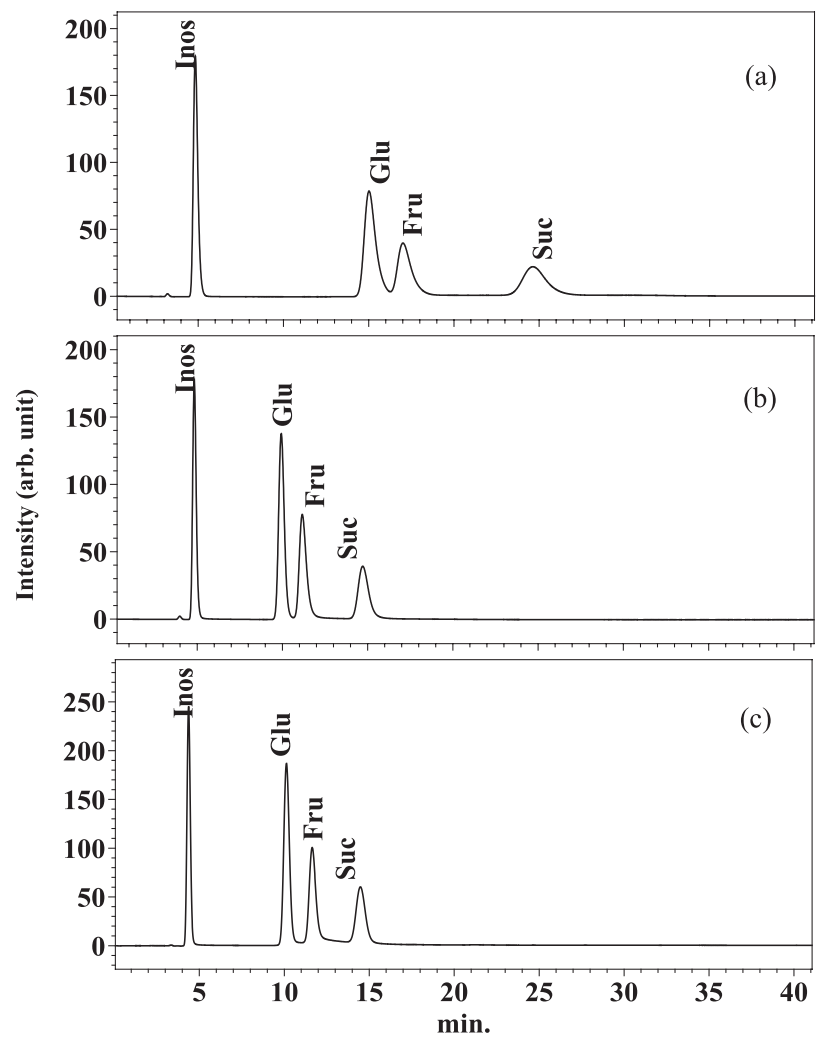

Fig. 5 Chromatograms obtained for separation of inositol, glucose, fructose, and sucrose using St-80 resin of (a) 10\%, (b) $40 \%$, and (c) $55 \%$ with $0.15 \mathrm{~mol} / \mathrm{L} \mathrm{NaOH}$ eluent at a flow rate of $0.5 \mathrm{~mL} / \mathrm{min}$.

We also examined the effect of the $0.15 \mathrm{~mol} / \mathrm{L} \mathrm{NaOH}$ eluent on the retention time. Chromatograms of St-80 (10\%), St-80 (40\%), and St-80 (55\%) obtained at a flow rate of $0.5 \mathrm{~mL} /$ min with $0.15 \mathrm{~mol} / \mathrm{L} \mathrm{NaOH}$ eluent are shown in Figs. 5a-c, respectively. Using $0.15 \mathrm{~mol} / \mathrm{L} \mathrm{NaOH}$ eluent, these resins showed good chromatography, similar to that of the fully porous resin. The retention times of sucrose for St-80 (10\%) with $0.15 \mathrm{~mol} / \mathrm{L} \mathrm{NaOH}$ eluent at flow rates of $0.3,0.5$, and $0.7 \mathrm{~mL} / \mathrm{min}$ were 41,25 , and $18 \mathrm{~min}$, respectively, which were all longer than those of St-80 (55\%).

The retention times of sucrose for St-80 (10\%), St-80 (40\%), and St-80 (55\%) with $0.10 \mathrm{~mol} / \mathrm{L} \mathrm{NaOH}$ eluent at a flow rate of $0.5 \mathrm{~mL} / \mathrm{min}$ were 46,21 , and $19 \mathrm{~min}$, respectively (Fig. 6). The retention time of sucrose for the fully porous resin was $27 \mathrm{~min}$. Thus, at all flow rates, the retention times of sucrose for both St-80 (55\%) and St-80 (40\%) were shorter than those of the fully porous resin. However, the retention time of sucrose for St-80 (10\%) was longer than that of the fully porous resin. This tendency was also observed at flow rates of 0.3 and $0.7 \mathrm{~mL} / \mathrm{min}$.

At a high $\mathrm{pH}$, the carbohydrates become more ionized, and consequently, their interaction with the porous layer

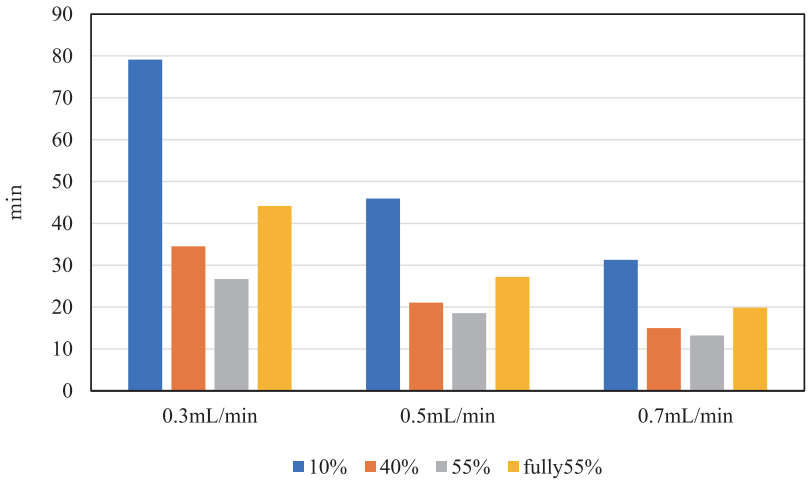

Fig. 6 Retention times of sucrose using St-80 (10\%, 40\%, and $55 \%)$ and fully porous resin (55\%) with $0.10 \mathrm{~mol} / \mathrm{L} \mathrm{NaOH}$ eluent at flow rates of $0.3,0.5$, and $0.7 \mathrm{~mL} / \mathrm{min}$.

increases. The elution sequence (in the order of glucose and then fructose) is the same as the $\mathrm{p} K_{\mathrm{a}}$ sequence (in the order of glucose and then fructose) ${ }^{22)}$.

The following aspects are important for understanding the remarkable separation properties of core-shell ion-exchange resins. First, the core suppresses solute diffusion, mainly along the column axis. Second, the moving distance of the solute within the shell is shortened because the porous layer is thin. Thus, the core-shell ion-exchange resins St-80 (55\%) and St-80 (40\%) exhibited shorter retention times than the fully porous resin.

It is considered that St-80 (10\%) provided a longer retention time than St-80 (40\%) because the ion-exchange capacities of St-80 (10\%) and St-80 (40\%) were $1.6 \mathrm{meq} / \mathrm{mg}$ and $1.2 \mathrm{meq} /$ $\mathrm{mg}$, respectively.

\subsection{Evaluation of theoretical plate number for St-80 (10\%), St-80 (40\%), and St-80 (55\%) ion-exchange resins}

We then evaluated the theoretical plate numbers $(N)$ of glucose, fructose, and sucrose in $0.10 \mathrm{~mol} / \mathrm{L} \mathrm{NaOH}$ eluent. At a flow rate of $0.5 \mathrm{~mL} / \mathrm{min}$, the values of $N$ of glucose for St-80 (10\%), St-80 (40\%), and St-80 (55\%) were 2460, 5060, and 4660, respectively (Fig. 7), whereas that for the fully porous resin was 5720. The values of $N$ for the core-shell ion-exchange resins were approximately $43 \%, 88 \%$, and $81 \%$, respectively, of that of the fully porous resin. The values of $N$ for St-80 (40\%) and St-80 (55\%) were slightly lower than that of the fully porous resin. By reducing the degree of cross-linking in the shell portion, the values of $N$ of glucose for St-80 (10\%) were low at this flow rate. By reducing the degree of cross-linking in the shell portion, a similar tendency was observed for the values of $N$ of glucose, fructose, and sucrose at flow rates of $0.3 \mathrm{~mL} / \mathrm{min}$ and $0.7 \mathrm{~mL} / \mathrm{min}$.

To examine the effect of varying the concentration of $\mathrm{NaOH}$ eluent on the value of $N$, a $0.15 \mathrm{~mol} / \mathrm{L} \mathrm{NaOH}$ eluent 


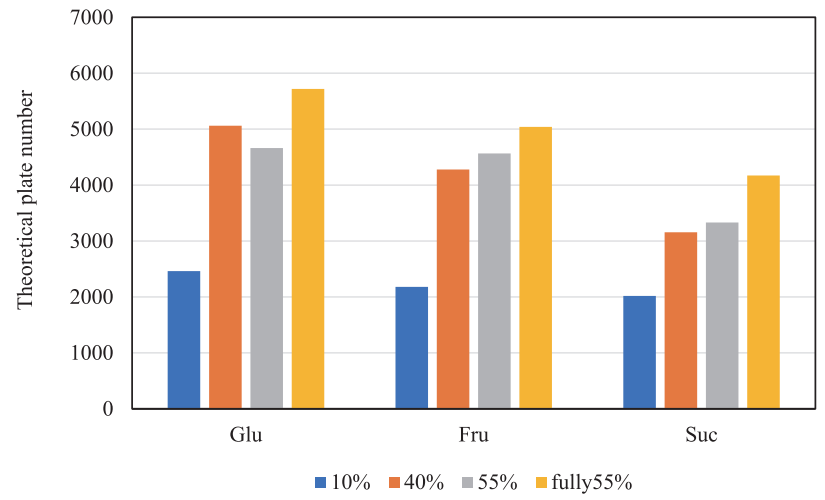

Fig. 7 Theoretical plate numbers of glucose, fructose, and sucrose for St-80 (10\%), St-80 (40\%), St-80 $(55 \%)$, and fully porous resin (55\%) with $0.10 \mathrm{~mol} / \mathrm{L} \mathrm{NaOH}$ eluent at a flow rate of $0.5 \mathrm{~mL} / \mathrm{min}$.

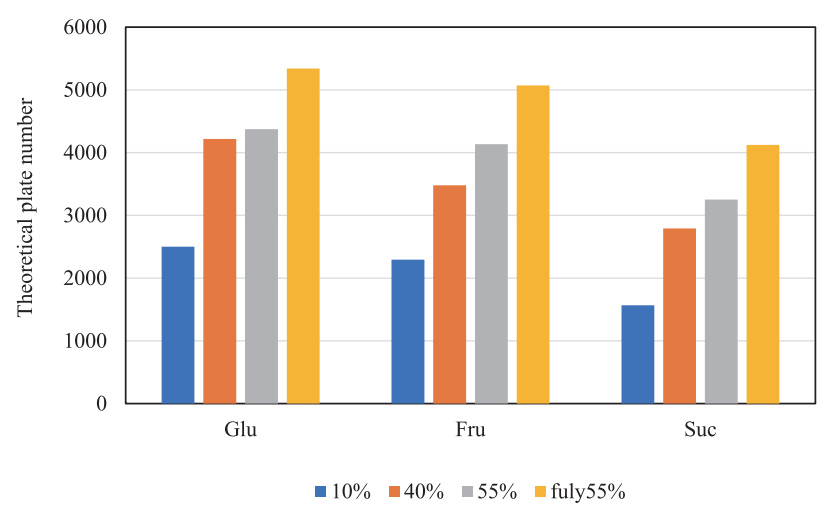

Fig. 8 Theoretical plate numbers of glucose, fructose, and sucrose for St-80 (10\%), St-80 (40\%), St-80 (55\%), and fully porous resin (55\%) with $0.15 \mathrm{~mol} / \mathrm{L} \mathrm{NaOH}$ eluent at a flow rate of $0.5 \mathrm{~mL} / \mathrm{min}$.

was used. The values of $N$ of glucose for St-80 (10\%), St-80 (40\%), and St-80 (55\%) were 2500, 4220, and 4370, respectively (Fig. 8), whereas that of the fully porous resin was 5340 .

The values of $N$ for St-80 (10\%), St-80 (40\%), and St-80 (55\%) were approximately $47 \%, 79 \%$, and $82 \%$, respectively, of that of the fully porous resin. The values of $N$ for St- 80
(40\%) and St-80 (55\%) were slightly lower than that of the fully porous resin at a flow rate of $0.5 \mathrm{~mL} / \mathrm{min}$.

By decreasing the degree of cross-linking in the shell portion, a similar tendency was observed for the values of $N$ of glucose, fructose, and sucrose at flow rates of $0.3 \mathrm{~mL} /$ min and $0.7 \mathrm{~mL} / \mathrm{min}$. Large values of $N$ for glucose, fructose, and sucrose were obtained when the core-shell ion-exchange resins had a high degree of cross-linking in the shell portion, such as $55 \%$.

The resins with degrees of cross-linking of $40 \%$ and $55 \%$ showed excellent separation, equal to that of the fully porous resin. Then, glucose, fructose, and sucrose showed large values of $N$ when the shell portion of the core-shell ion-exchange resin was fabricated with a high degree of cross-linking.

\subsection{Durability of the series of St-80 resins}

The effect of the usage period on the performance of St-80 $(10 \%, 40 \%$, and $55 \%)$ was then investigated by comparing the retention time of sucrose, resolution between glucose and fructose (Table 2), and value of $N$ of glucose, fructose, and sucrose. These values for St-80 (10\%), St-80 (40\%), and St-80 (55\%) were obtained after 150 (in 19 months) and 230 (in 17 months), and 845 (in 21 months) times of measurement, respectively.

For St-80 (10\%) and St-80 (40\%), the retention time and resolution between glucose and fructose were almost unchanged (Table 2), and the values of $N$ of glucose, fructose, and sucrose remained at $\sim 90 \%$ compared with those of the newly prepared resin.

For St-80 (55\%), on the other hand, the retention times were almost the same, but the resolution decreased gradually from 2.2 to 1.8 (Table 2). The values of $N$ of those for St- 80 (55\%) decreased to $74 \%, 69 \%$, and $75 \%$, respectively.

\section{Conclusion}

In this study, we evaluated core-shell ion-exchange resins with cross-linking degrees of $10 \%, 40 \%$, and $55 \%$ in the porous portion of St-80. When the retention times of various

Table 2 Effect of the number of measuring times (period) for St-80 (10\%, 40\%, and $55 \%$ ) on the retention times of sucrose, resolution between glucose and fructose, and values of $N$ of glucose, fructose, and sucrose. Elution condition: $0.10 \mathrm{~mol} / \mathrm{L} \mathrm{NaOH}$ eluent, $0.5 \mathrm{~mL} / \mathrm{min}$

\begin{tabular}{llccccc}
\hline & $\begin{array}{l}\text { Number of times } \\
\text { (period) }\end{array}$ & RT of Suc & Resolution & \multicolumn{2}{c}{$N$ of Glu, Fru, and Suc } \\
\hline \multirow{2}{*}{ St-80 (10\%) } & initial & 45.9 & 1.9 & 2460 & 2180 & 2020 \\
& 150 (19 months) & 45.8 & 1.9 & 2250 & 2320 & 1790 \\
\hline \multirow{2}{*}{ St-80 (40\%) } & initial & 21.1 & 2.2 & 5060 & 4280 & 3160 \\
& 230 (17 months) & 21.2 & 2.3 & 4450 & 3590 & 3220 \\
\hline \multirow{2}{*}{ St-80 (55\%) } & initial & 18.6 & 2.2 & 4660 & 4570 & 3330 \\
& 845 (21 months) & 18.5 & 1.8 & 3430 & 3140 & 2500 \\
\hline
\end{tabular}


carbohydrates were compared, they decreased with an increasing degree of cross-linking in the shell layer. In other words, St-80 (40\%) and St-80 (55\%) showed shorter retention times than the fully porous resin. Moreover, St-80 (40\%) and St-80 (55\%) showed larger theoretical plate numbers $(N)$ than St-80 (10\%). Thus, St-80 (40\%) and St-80 (55\%) are highly efficient core-shell ion-exchange resins for carbohydrate analysis.

Since previously reported core-shell ion-exchange resins can be used under strong alkaline conditions, the resins described herein are effective for the analysis of carbohydrates using ECD. Furthermore, these resins have outstanding durability; they show similar analytical performance even after exceeding 17 months of use.

Notably, these resins can be applied to other fields, such as in the analysis of foods and pharmaceuticals, by introducing different functional groups and controlling the pore size in the shell portion.

\section{Acknowledgement}

We thank Prof. Nobuharu Takai for valuable discussions.

\section{References}

1) J. J. Kirkland, Anal. Chem., 64, 1239 (1992).

2) J. J. Kirkland, T. J. Langlois and J. J. DeStefano, Amer. Lab., 39, 18 (2007).

3) N. Nagae, T. Enami and S. Doshi, LCGC NORTH AMERICA, 20, 964 (2002).

4) J. Ruta, D. Zurlino, C. Grivel, S. Heinisch, J, Veuthey and D. Guillarme, J. Chromatogr. A, 1228, 221 (2012).

5) A. Ahmed, W. Abdelmagid, H. Ritchie, P. Myers and H. Zhang, J. Chromatogr. A, 1274, 194 (2012).

6) N. Nagae, T. Tsukamoto and V. D. Gaitonde, CHROMATOGRAPHY TODAY, 8, 18 (2015).

7) R. Aljhni, C. Andre, L. Lethier and Y. C. Guillaume, Talanta, 144, 226 (2015)

8) X. Zhao, H. Zhang, X. Zhou, L. Wang, L. Wan and R. Wu, Chinese Journal of Chromatography, 38(12), 1357 (2020).

9) S. Podzimek, Chromatographia, 33, 377 (1992).

10) Y. C. Lee, J. Chromatogr. A, 720, 137 (1996).

11) T. R. I. Cataldi, C. Campa and G. E. De Benedetto, J. Anal.
Chem., 368, 739 (2000).

12) K. Inoue, K. Yamazaki, K. Kitahara, Y. Aikawa, S. Arai and T. Hanada-Masuda, Bunseki Kagaku, 60(12), 959 (2011) (in Japanise).

13) W. Li and H. D. H. Stöver, Macromolecules, 33, 4354 (2000).

14) F. Bai, X. Yang and W. Huang, Macromolecules, 37, 9746 (2004).

15) Showa Denko K.K., Japanese patent. 4979059 (in Japanese).

16) N. Takashi, E. Fukushi, S. Onodera, T. Nishimoto, J. Kawabata and N. Shiomi, J. Appl. Glycosci., 54, 195 (2007).

17) G. Pfeiffer, H. Geyer, R. Geyer, I. Kalsner and P. Wendorf, Biomed. Chromatogr., 4, 193 (1990).

18) A. Miyashita, M. Usui and N. Takai, Japanese patent 6218574 (in Japanese).

19) T. Masuda, Y. Nishimura, M. Tonegawa, K. Kitahara, S. Arai, J. Yamashita and N. Takai, Chem. Lett., 26, 1239 (1997).

20) T. Masuda, Y. Nishimura, M. Tonegawa, K. Kitahara, S. Arai, J. Yamashita and N. Takai, J. Chromatogr. A, 845, 401 (1999).

21) T. Masuda, K. Kitahara, Y. Aikawa and S. Arai, Anal. Sci., 17, i1895 (2001).

22) T. Masuda, K. Kitahara, Y. Aikawa and S. Arai, J. Chromatogr. A, 961, 89 (2002).

23) K. Kitahara, S. Okuya, I. Yoshihama, T. Hanada, K. Nagashima and S. Arai, J. Chromatogr. A, 1216, 7409 (2009).

24) K. Inoue, K. Kitahara, Y. Aikawa, S. Arai and T. Masuda-Hanada, Molecules, 16(7), 5905 (2011).

25) S. Mitomo, Y. Negishi and T. Mutai, the 66th Annual Convention of Japan Society for Analytical Chemistry, Tokyo Japan, 378, 2017 (in Japanese).

26) S. Mitomo, Y. Negishi and T. Mutai, the 30th Biomedical Analytical Science Symposium, Tokyo Japan, 102, 2017 (in Japanese).

27) S. Mitomo and Y. Negishi, the 97th Annual Meeting of The Chemical Society of Japan, Kanagawa Japan, 1PB-149, 2017 (in Japanese).

28) Y. Inoue, N. Komiya, I. Murata, S. Mitomo, Y. Negishi and I. Kanamoto, J. Drug Res. Dev., 3(3) (2017)

29) Y. Inoue, A. Mitumura, S. Narumi, I. Murata, S. Mitomo, Y. Negishi and I. Kanamoto, World J. Pharm. Sci., 6(2), 47 (2018).

30) S. Mitomo, Y. Negishi, T. Mutai and Y. Inoue, J. Life Support Eng., 31(4) 158 (2019).

31) The Japanese Pharmacopoeia, 31(4) (in Japanese). 